\title{
Problems With Diagnosis by Fluid-Attenuated Inversion Recovery Magnetic Resonance Imaging in Patients With Acute Aneurysmal Subarachnoid Hemorrhage
}

\author{
Masami SHIMODA, Kaori HoSHIKAWA, Hideki SHIRAMIZU, \\ Shinri ODA, and Mitsunori MATSUMAE*
}

Department of Neurosurgery, Tokai University Hachioji Hospital, Hachioji, Tokyo; *Department of Neurosurgery, Tokai University School of Medicine, Isehara, Kanagawa

\begin{abstract}
The diagnostic efficacy of fluid-attenuated inversion recovery (FLAIR) magnetic resonance imaging and computed tomography (CT) for acute subarachnoid hemorrhage (SAH) were compared and the problems with diagnosis were investigated in 81 patients with aneurysmal SAH within 24 hours after onset who underwent FLAIR imaging and CT on admission. The number of hematomas in the cisterns and ventricles were evaluated by clot scores. In addition, the frequency of undetected hematomas was calculated for the cisterns and ventricles. Clot scores were significantly higher for FLAIR imaging than for $\mathrm{CT}$ in the lateral sylvian, quadrigeminal, and convexity cisterns. On the other hand, clot scores were significantly higher for CT than for FLAIR imaging in the interhemispheric and medial sylvian cisterns. The overall frequency of undetected SAH was $2 \%$ for FLAIR imaging and $14 \%$ for CT. With the exception of the interhemispheric and medial sylvian cisterns, the frequency of undetected $\mathrm{SAH}$ was higher for CT than for FLAIR imaging. In this study, FLAIR imaging was more sensitive than CT for the detection of acute $\mathrm{SAH}$ within 24 hours after onset. However, the diagnostic efficacy of FLAIR imaging was reduced in comparatively tight cisterns.
\end{abstract}

Key words: acute stage, fluid-attenuated inversion recovery magnetic resonance imaging, intraventricular hemorrhage, subarachnoid hemorrhage

\section{Introduction}

Computed tomography (CT) has been the first choice for neuroradiological imaging of patients with stroke or sudden onset of acute headache. However, some headaches are caused by pathologic conditions that cannot be detected by CT. In particular, magnetic resonance (MR) imaging is often necessary to determine the primary cause of the headaches in patients with thunderclap headache, ${ }^{5)}$ reversible cerebral vasoconstriction syndrome, ${ }^{7)}$ or cerebral venous thrombosis. Rapid and accurate diagnosis of acute-phase cerebral infarctions depends on MR imaging as the first choice in more and more stroke centers, so neurosurgeons often have substantial experience with the MR imaging findings of the acute phase of subarachnoid hemorrhage (SAH). However, MR imaging is generally accepted as less informative than CT for the detection of acute SAH.

Received October 22, 2009; Accepted January 13, 2010
Several recent studies have reported that fluid-attenuated inversion recovery (FLAIR) MR imaging provides reliable diagnostic images of acute or subacute SAH. ${ }^{14,15,18,19)}$ FLAIR MR imaging uses an inversion-recovery pulse sequence with an inversion time that effectively nullifies the signal from cerebrospinal fluid (CSF) and provides heavy $\mathrm{T}_{2}$ weighting due to its long echo time. This technique is useful for the evaluation of diseases affecting the subarachnoid space such as $\mathrm{SAH}$ and meningeal diseases including meningitis. ${ }^{14,15,18,25)}$ However, few neurosurgeons use FLAIR imaging as the first choice for the diagnosis of SAH, primarily because of the poor understanding of the differences in diagnostic characteristics between FLAIR imaging and CT for SAH. In addition, the potential false-negative findings at the time of diagnosis of acute SAH using FLAIR imaging are not well characterized.

The present study compared the findings of FLAIR MR imaging and CT in patients with acutephase SAH to investigate the problems with SAH diagnosis by FLAIR imaging as the first-choice neuro- 
imaging method.

\section{Materials and Methods}

A total of 107 patients with nontraumatic aneurysmal SAH were admitted to Tokai University Hachioji Hospital within 24 hours after onset between September 2002 and December 2008. CT and MR imaging were performed together in patients with SAH and stable vital signs reflecting adequate respiration and circulation, after obtaining informed consent from the patient's family based on the rules of examination established by the Clinical Manual of Tokai University Hachioji Hospital. The 107 patients included 81 patients without rerupture who underwent both FLAIR imaging and CT at the time of admission and were analyzed retrospectively.

On admission, MR imaging including axial conventional FLAIR imaging and MR angiography was performed using a 1.5-Tesla superconducting magnet (Signa EXCITE; General Electric Medical Systems, Milwaukee, Wis., U.S.A.) with a quadrature head coil. FLAIR imaging used the following parameters: repetition time, $8000 \mathrm{msec}$; inversion time, $2000 \mathrm{msec}$; echo time, $135 \mathrm{msec}$; size of field of view, $240 \mathrm{~mm}$; imaging matrix, $256 \times 224$; section gap, $1.0 \mathrm{~mm}$; number of excitations, 1 ; and thickness, $5 \mathrm{~mm}$. MR angiography was simultaneously performed to search for the SAH source and identify congruity between the site of the aneurysm and localization of the SAH clot. All MR imaging was completed within 13-15 minutes. CT was performed almost simultaneously with MR imaging. CT slice thickness was $4 \mathrm{~mm}$. Multi-detector row CT images were acquired using a 4-mm collimated beam with the following characteristics: gantry rotation time, $1.5 \mathrm{sec}$; $\mathrm{x}$-ray tube voltage, $120 \mathrm{kV}$; tube current, 250 $\mathrm{mA}$; and total scan time, 50 sec.

$\mathrm{CT}$ and MR imaging findings were interpreted by at least two experienced senior neurosurgeons. The clinical grade of each patient was determined on admission according to the World Federation of Neurological Surgeons system. ${ }^{6)}$ Patients in grade 4 or 5 were defined as having low grade SAH. The severity of SAH was classified radiologically using CT according to Fisher's scale. ${ }^{8)}$

SAH observed on CT and FLAIR imaging was scored semiquantitatively as the sum of clot scores ranging from 0 to 3 for each of the 10 cisterns: 0 , no blood; 1, small amount of blood; 2, moderate amount of blood; and 3, completely filled with blood. ${ }^{10)} \mathrm{We}$ added analysis of the bilateral convexity sulci to the original grading of subarachnoid clot, so a total of 12 cisterns were investigated. These clot scores were added to yield an SAH sum score ranging from 0 to
36. The amount of intraventricular hemorrhage (IVH) in the lateral, third, and fourth ventricles was scored as follows: 0 , no blood; 1 , sedimentation of blood in the posterior part; 2, partly filled with blood; and 3, completely filled with blood. The total clot score of IVH was the sum of the four scores and ranged from 0 to $12 .{ }^{10,12)}$ Analysis of intracerebral hemorrhage or intrasylvian hematoma was based on subarachnoid clots and intraparenchymal hemorrhage (more than $10 \mathrm{ml}$ ) detected by CT. Hydrocephalus was evaluated by measuring the bicaudate index and the temporal horn diameter. ${ }^{26)}$ Undetected SAH or IVH in each cistern or ventricle was defined as clot undetected by one technique (i.e., FLAIR imaging or CT) but detected by the other.

The Wilcoxon matched pairs signed ranks test was used to analyze differences in SAH or IVH scores between CT and FLAIR imaging within each cistern or ventricle. In addition, the frequency of absence of SAH was calculated. All statistical analyses were performed using a commercially available software program (SPSS ver. 16.0 for Windows; Mehta and Patel/SPSS, Chicago, Ill., U.S.A.).

\section{Results}

The clinical features of the 81 patients with SAH who underwent FLAIR imaging and CT on admission are shown in Table 1. Initial MR imaging including FLAIR was conducted within 6 hours of $\mathrm{SAH}$ onset in 70 patients. Adverse events due to MR imaging did not occur in any patient. One patient with negative findings on CT and FLAIR imaging was given a diagnosis of SAH following lumbar puncture based on the MR angiography findings suggesting ruptured aneurysm. No diagnosis of SAH was based on only the FLAIR imaging or CT findings, despite the absence of clot on either CT or FLAIR imaging. MR angiography could not identify the hemorrhage source in 5 of the 81 cases. The hemorrhage source was identified by conventional angiography in these cases. Anterior wall aneurysms of the internal carotid artery were identified in three cases, a dissecting aneurysm of anterior cerebral artery in one case, and a middle cerebral artery aneurysm with severe atherosclerosis in one case.

The SAH and IVH clot scores determined by CT and FLAIR imaging are summarized in Table 2. SAH clot scores on FLAIR imaging were significantly $(\mathrm{p}<0.001)$ higher than scores on CT in the lateral sylvian, quadrigeminal, and convexity cisterns. On the other hand, SAH clot scores on CT were significantly higher $(\mathrm{p}<0.001)$ in the interhemispheric and medial sylvian cisterns. IVH clot scores on 
Table 1 Clinical features of 81 patients on admission

\begin{tabular}{|c|c|}
\hline \multicolumn{2}{|l|}{ Age (yrs) } \\
\hline mean \pm standard deviation & $57.8 \pm 13.1$ \\
\hline range & $21-78$ \\
\hline Elderly patients ( $\geq 70$ yrs) & $19(23 \%)$ \\
\hline Female & $48(59 \%)$ \\
\hline \multicolumn{2}{|l|}{ WFNS grade on admission } \\
\hline I & $37(46 \%)$ \\
\hline II & $14(17 \%)$ \\
\hline IV & $13(16 \%)$ \\
\hline $\mathrm{V}$ & $17(21 \%)$ \\
\hline \multicolumn{2}{|l|}{ Fisher group } \\
\hline 1 & $1(1 \%)$ \\
\hline 2 & $15(19 \%)$ \\
\hline 3 & $55(68 \%)$ \\
\hline 4 & $10(12 \%)$ \\
\hline Acute hydrocephalus & $36(44 \%)$ \\
\hline \multicolumn{2}{|l|}{ MR imaging timing } \\
\hline mean \pm standard deviation (hrs) & $4.2 \pm 4.8$ \\
\hline within $3 \mathrm{hrs}$ of SAH onset & $55(68 \%)$ \\
\hline 4-6 hrs after SAH onset & $15(19 \%)$ \\
\hline 7-12 hrs after SAH onset & $6(7 \%)$ \\
\hline $13-24$ hrs after SAH onset & $5(6 \%)$ \\
\hline \multicolumn{2}{|l|}{ Ruptured aneurysm site } \\
\hline anterior communicating artery & $28(35 \%)$ \\
\hline anterior cerebral artery & $2(2 \%)$ \\
\hline internal carotid artery & $26(32 \%)$ \\
\hline middle cerebral artery & $18(22 \%)$ \\
\hline vertebrobasilar system & $7(9 \%)^{*}$ \\
\hline
\end{tabular}

Values are n (\%) unless otherwise indicated. *Includes two cases of dissecting aneurysm and three cases of distal posterior inferior cerebellar artery aneurysm. MR: magnetic resonance, SAH: subarachnoid hemorrhage, WFNS: World Federation of Neurological Surgeons.

FLAIR imaging were significantly $(p=0.011)$ higher than scores on $\mathrm{CT}$ in the lateral ventricle. There were no other significant differences in SAH or IVH clot scores in other ventricles or cisterns.

Frequencies of SAH undetected by FLAIR imaging and CT are summarized in Table 3. The frequency of SAH undetected by FLAIR imaging (only CT-detected) was higher than that undetected by CT (only FLAIR-detected) in the interhemispheric cistern. The frequency of SAH undetected by CT (only FLAIR-detected) was higher than that undetected by FLAIR imaging (only CT-detected) in the lateral sylvian fissure and the suprasellar, ambient, quadrigeminal, and convexity cisterns. The frequencies of undetected SAH were similar for CT and FLAIR imaging in the medial sylvian fissure. Typical cases are shown in Figs. 1-3.

Frequencies of IVH undetected by FLAIR imaging and CT are shown in Table 4. The frequency of IVH undetected by CT was higher than that undetected
Table 2 Differences in subarachnoid hemorrhage (SAH) or intraventricular hemorrhage (IVH) clot scores between computed tomography (CT) and fluid-attenuated inversion recovery (FLAIR) magnetic resonance imaging

\begin{tabular}{lccccc}
\hline \multirow{2}{*}{$\begin{array}{c}\text { Site of cistern } \\
\text { or ventricle }\end{array}$} & \multicolumn{2}{c}{ Mean SAH point } & & Difference CT-FLAIR \\
\cline { 2 - 3 } \cline { 6 - 7 } \cline { 6 - 7 } & CT & FLAIR & & Z & p Value \\
\hline Interhemispheric cistern & 1.31 & 0.97 & & +3.801 & $<0.001$ \\
Lateral sylvian cistern & 1.50 & 1.67 & & -3.357 & $<0.001$ \\
Medial sylvian cistern & 1.70 & 1.39 & & +4.306 & $<0.001$ \\
Suprasellar cistern & 1.71 & 1.65 & & +0.825 & 0.409 \\
Ambient cistern & 1.32 & 1.25 & & +0.642 & 0.521 \\
Quadrigeminal cistern & 0.95 & 1.53 & & -5.346 & $<0.001$ \\
Convexity cistern & 1.01 & 1.28 & & -6.508 & $<0.001$ \\
\hline \multicolumn{1}{c}{ Total SAH points } & 12.76 & 12.92 & & -1.802 & 0.072 \\
\hline Third ventricle & 0.80 & 0.91 & & -1.098 & 0.272 \\
Fourth ventricle & 0.80 & 0.86 & & -0.708 & 0.479 \\
Lateral ventricle & 0.34 & 0.47 & -2.552 & 0.011 \\
\hline \multicolumn{1}{c}{ Total IVH points } & 5.66 & 5.86 & -1.906 & 0.057 \\
\hline
\end{tabular}

Statistical differences were determined by Wilcoxon matched pairs signed ranks test.

Table 3 Frequency of undetected subarachnoid hemorrhage (SAH) on admission

\begin{tabular}{lrccc}
\hline Site of cistern & $\begin{array}{c}\text { Total } \\
\text { No. of } \\
\text { sites }\end{array}$ & $\begin{array}{c}\text { CT- or } \\
\text { FLAIR- } \\
\text { detected } \\
\text { SAH }\end{array}$ & $\begin{array}{c}\text { FLAIR- } \\
\text { undetected } \\
\text { (only CT- } \\
\text { detected) } \\
\text { SAH }\end{array}$ & $\begin{array}{c}\text { CT- } \\
\text { undetected } \\
\text { (only } \\
\text { FLAIR- } \\
\text { detected) } \\
\text { SAH }\end{array}$ \\
\hline Interhemispheric & 81 & 62 & $11(18 \%)$ & $2(3 \%)$ \\
Lateral sylvian & 162 & 132 & 0 & $20(15 \%)$ \\
Medial sylvian & 162 & 141 & $10(7 \%)$ & $9(6 \%)$ \\
Suprasellar & 162 & 134 & $2(1 \%)$ & $13(10 \%)$ \\
Ambient & 162 & 129 & $5(4 \%)$ & $15(12 \%)$ \\
Quadrigeminal & 81 & 65 & 0 & $20(31 \%)$ \\
Convexity & 162 & 109 & $2(2 \%)$ & $30(28 \%)$ \\
\hline \multicolumn{1}{c}{ Total } & 972 & 772 & $19(2 \%)$ & $109(14 \%)$ \\
\hline
\end{tabular}

Statistical significance of the frequency of CT- or FLAIRundetected SAH was not calculated because no control subjects without SAH were included in the study. CT: computed tomography, FLAIR: fluid-attenuated inversion recovery magnetic resonance imaging.

by FLAIR imaging in the third and lateral ventricles. The frequencies of IVH undetected by CT or FLAIR imaging were similar in the fourth ventricle.

Cisternal SAH in the posterior fossa was depicted more clearly on FLAIR imaging than on CT in the seven patients with ruptured posterior circulation aneurysm. FLAIR imaging detected not only fourth ventricle IVH but also cisternal SAH in the posterior fossa in three patients with ruptured distal posterior 

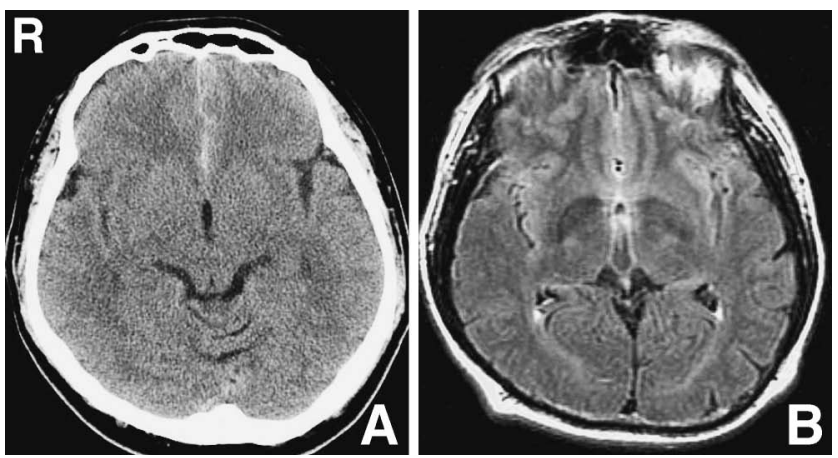

Fig. 1 A 43-year-old man with hyperacute subarachnoid hemorrhage caused by a ruptured anterior communicating artery aneurysm. Neuroradiological images were obtained on admission at 4 hours after onset. A: Computed tomography scan showing a thin but apparent subarachnoid clot in the interhemispheric cistern. B: Fluid-attenuated inversion recovery magnetic resonance image showing no apparent subarachnoid hemorrhage in the interhemispheric cistern.
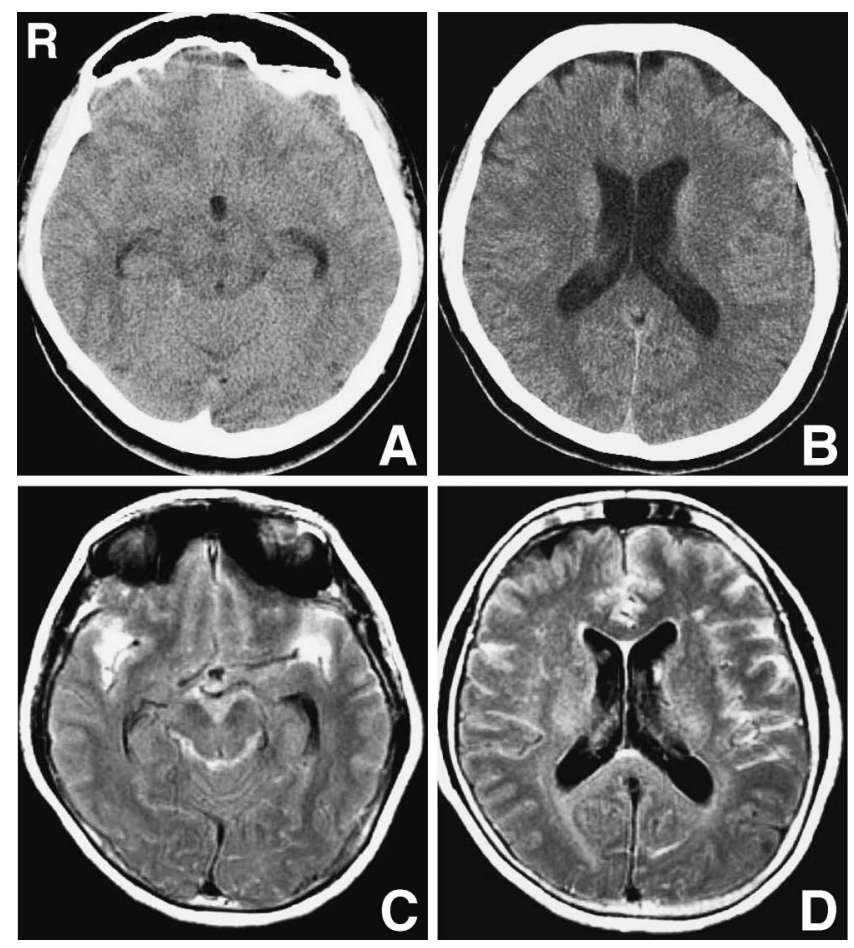

Fig. 2 A 61-year-old man with hyperacute subarachnoid hemorrhage caused by a ruptured right internal carotid artery aneurysm. Neuroradiological images were obtained on admission at 2 hours after onset. A, B: Computed tomography scans showing no apparent subarachnoid hemorrhage in the lateral sylvian and convexity cisterns. C, D: Fluid-attenuated inversion recovery magnetic resonance images showing thick subarachnoid hemorrhage in the bilateral lateral sylvian and convexity cisterns.
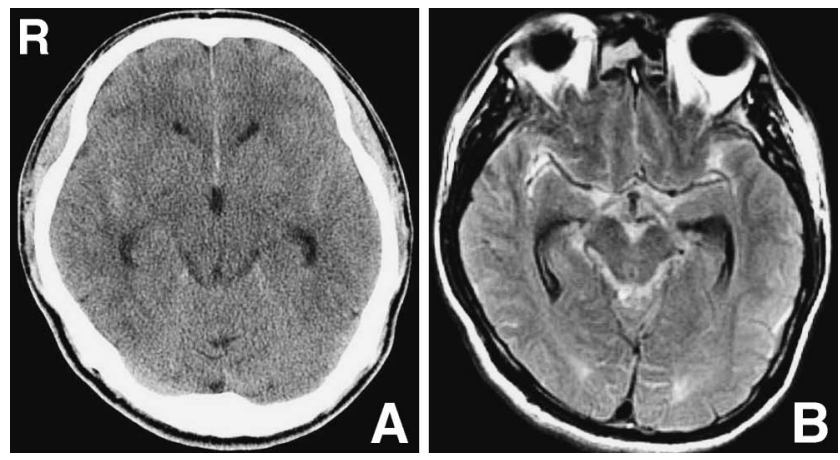

Fig. 3 A 39-year-old man with hyperacute subarachnoid hemorrhage caused by a ruptured anterior communicating artery aneurysm. Neuroradiological images were obtained on admission at 2 hours after onset. A: Computed tomography scan showing a subarachnoid clot in the interhemispheric cistern, but no apparent subarachnoid hemorrhage in the quadrigeminal cistern. B: Fluid-attenuated inversion recovery magnetic resonance image showing thick subarachnoid hemorrhage in the quadrigeminal cistern, but no apparent subarachnoid hemorrhage in the interhemispheric cistern.

Table 4 Frequency of undetected intraventricular hemorrhage (IVH) on admission

\begin{tabular}{lcccc}
\hline $\begin{array}{c}\text { Site of } \\
\text { ventricle }\end{array}$ & $\begin{array}{c}\text { Total } \\
\text { No. of } \\
\text { sites }\end{array}$ & $\begin{array}{c}\text { CT- or } \\
\text { FLAIR- } \\
\text { detected } \\
\text { IVH }\end{array}$ & $\begin{array}{c}\text { FLAIR- } \\
\text { undetected } \\
\text { (only CT- } \\
\text { detected) } \\
\text { IVH }\end{array}$ & $\begin{array}{c}\text { CT- } \\
\text { undetected } \\
\text { (only } \\
\text { FLAIR- } \\
\text { detected) } \\
\text { IVH }\end{array}$ \\
\hline Third & 81 & 38 & 4 & 8 \\
Fourth & 81 & 37 & 4 & 5 \\
Lateral & 162 & 50 & 7 & 18 \\
\hline \multicolumn{1}{c}{ Total } & 324 & 125 & 15 & 31 \\
\hline
\end{tabular}

Statistical significance of the frequency of CT- or FLAIRundetected IVH was not calculated because no control subjects without subarachnoid hemorrhage were included in the study. CT: computed tomography, FLAIR: fluid-attenuated inversion recovery magnetic resonance imaging.

inferior cerebellar artery (PICA) aneurysm (Fig. 4A, B). MR angiography confirmed ruptured aneurysm of the distal PICA (Fig. 4C).

\section{Discussion}

CT was previously considered to be the gold standard for the diagnosis of SAH. However, CT is unreliable for detecting subarachnoid blood produced by a minor leak and the diagnosis rate of small SAH is not acceptable. ${ }^{11,22,24,27)}$ Unfortunately, if a minor leak is unrecognized and subsequent major rupture 

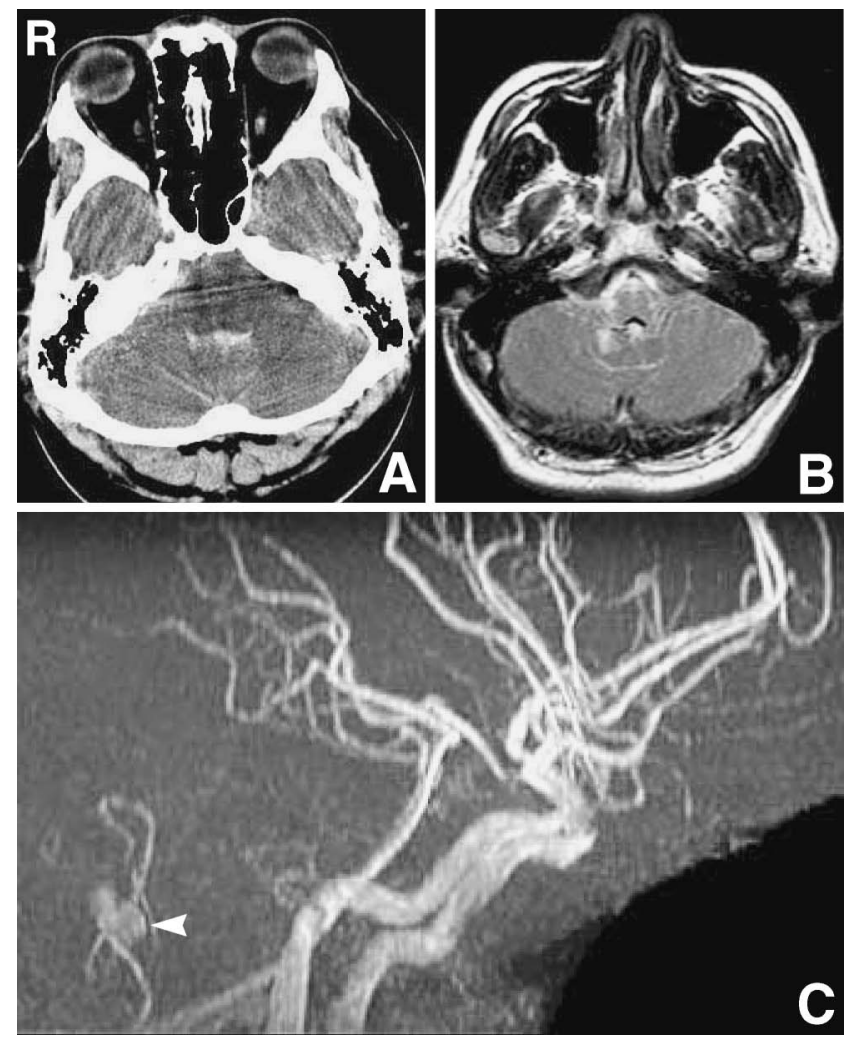

Fig. 4 A 41-year-old man with hyperacute subarachnoid hemorrhage caused by a ruptured distal posterior inferior cerebellar artery aneurysm. Neuroradiological images were obtained on admission at 3 hours after onset. A: Computed tomography scan showing fourth ventricule intraventricular hemorrhage, but no apparent cisternal subarachnoid hemorrhage in the posterior fossa due to beam-hardening artifacts. B: Fluid-attenuated inversion recovery magnetic resonance image showing both fourth ventricule intraventricular hemorrhage and cisternal subarachnoid hemorrhage in the posterior fossa without artifacts. C: Magnetic resonance angiogram showing an aneurysm in the distal posterior inferior cerebellar artery (arrowhead).

occurs, the SAH is more severe and the clinical condition of the patient becomes more precarious.

CT detection of SAH depends on the attenuation values of blood in the CSF spaces, which are linearly related to the hematocrit and hemoglobin levels. ${ }^{21)}$ However, CT is not sensitive for detecting pathological changes in CSF proteins. ${ }^{21)}$ In contrast, FLAIR imaging responds to increases in protein or cellularity in the CSF and can detect mixtures of blood or exudates in the CSF in patients with SAH or bacterial or cancer-related meningitis. ${ }^{11,14,25)}$ Experimental studies have shown that small amounts of acute SAH diluted by CSF are more readily identified with FLAIR imaging than with CT, and that
FLAIR imaging is more sensitive than CT for the detection of acute SAH. ${ }^{1,20,28)}$ The clinical sensitivity of SAH detection by FLAIR imaging is higher than that of CT in both the subacute stage of SAH, when the diagnostic ability of CT decreases, and the acute phase of SAH. ${ }^{14,15,18,19)}$

In our study, FLAIR imaging was more sensitive than CT for the detection of acute SAH within 24 hours after onset. In addition, the overall frequency of undetected acute SAH was higher for CT than for FLAIR imaging. These findings are consistent with current clinical experience, which suggests that FLAIR imaging is more sensitive for the diagnosis of acute SAH.

Furthermore, our results suggest that lumbar puncture is not necessary if SAH is identified only by FLAIR imaging, without confirmation of subarachnoid clot by CT. However, if MR angiography fails to reveal the source of the hemorrhage, and SAH is simultaneously associated with apparent inflammation findings such as pyrexia, examination of the CSF is necessary. Therefore, FLAIR imaging can distinguish meningitis mimicking $\mathrm{SAH}$ from aneurysmal or other vascular SAH.

FLAIR imaging findings were positive in only 2 of 12 patients with SAH revealed by lumbar puncture but negative CT findings, suggesting that despite the sensitivity of FLAIR for SAH, lumbar puncture is still indispensable in the work-up of a patient with suspected SAH. ${ }^{16)}$ Aneurysmal SAH occurred in only 5 of the 12 cases. Therefore, FLAIR imaging has important diagnostic limits in patients with minor hemorrhages, such as non-aneurysmal SAH. In the present study, all cases were aneurysmal SAH. Neither CT nor FLAIR imaging identified SAH in one case. Lumbar puncture was performed based on the sudden onset of headache, and MR angiography identified an aneurysm. Therefore, we did not overlook any diagnosis of SAH in a patient with minor hemorrhage. However, this potential limitation of FLAIR imaging shows that MR angiography should be simultaneously performed to determine the hemorrhagic source.

The diagnostic sensitivity of CT and FLAIR imaging varied by cistern. The SAH clot score and frequency of undetected SAH were higher for CT than FLAIR imaging in cisterns with comparatively wide CSF spaces. In contrast, the SAH clot score and frequency of undetected SAH were higher for FLAIR imaging than CT in cisterns with comparatively tight CSF spaces. These results suggest that the diagnostic sensitivity of FLAIR imaging for the detection of acute SAH was reduced in comparatively tight cisterns, perhaps because of the low signal intensity associated with the flow void of arteries in the 
cisterns.

Clinicians need to keep in mind that CSF hyperintense artifacts can mimic the appearance of SAH or IVH on FLAIR imaging. ${ }^{14)}$ Such artifacts are especially prominent in the ventricular system and cisterns around the basilar artery, resulting from head motion of the patient ${ }^{4)}$ and/or CSF pulsation. ${ }^{2,3,9,14,29)}$ CSF pulsation artifacts are attributable to ghosting artifacts caused by the inflow of non-inverted (and hence non-nullified) CSF into sections with high CSF flow rate. ${ }^{2,14,29)}$ Such artifacts appear as SAH-like irregular hyperintensity and are difficult to differentiate from subarachnoid clots on FLAIR imaging. However, this CSF pulsation artifact almost always occurs in the isolated cisterns around a basilar artery, such as the prepontine cistern. Therefore, if an irregular hyperintensity is confined to the prepontine cistern, and MR angiography confirms absence of abnormality of the basilar artery, the possibility of a CSF artifact is high.

CSF pulsation artifact is an important drawback of FLAIR imaging in ventricular systems, and is commonly found in the third and fourth ventricles. ${ }^{2)}$ Ventricular CSF artifacts are common in adults of all ages, but are associated with advancing age and increasing ventricular size. ${ }^{2)}$ Furthermore, ventricular CSF artifacts might obscure or mimic intraventricular lesions, particularly in the third and fourth ventricles. ${ }^{2,3)}$ Recently, the use of interleaved acquisition has been proposed to eliminate CSF pulsation and motion artifacts. ${ }^{4,9,14,29)}$ We hope that routine FLAIR imaging can be established to eliminate these artifacts.

The compromise of FLAIR imaging for detecting third and fourth ventricule IVH by CSF pulsation artifacts $^{2,3)}$ indicate that care is required for the diagnosis of only IVH without cisternal SAH, because a false diagnosis can create high anxiety in the patient. In particular, primary or idiopathic $\mathrm{IVH}^{23)}$ localized in the third or fourth ventricle is difficult to diagnose only by FLAIR imaging. Future clinical investigations of MR imaging findings in patients with primary IVH are necessary.

In contrast, the differential diagnosis of true IVH and CSF pulsation artifact is comparatively easy in the lateral ventricle, because CSF pulsation artifact in the lateral ventricle frequently appears on FLAIR imaging in the bilateral frontal horns located in the foramen of Monro. Therefore, any hyperintensity in the lateral ventricle other than in the frontal horn above the foramen of Monro is most likely to be true IVH. Although CT may still be necessary to exclude IVH, we believe that FLAIR imaging is at least as useful as CT for identifying acute lateral ventricle
IVH.

CT findings of isolated fourth ventricle IVH without cisternal SAH are important in the diagnosis of ruptured distal PICA aneurysm. ${ }^{13)}$ However, we found that cisternal SAH in the posterior fossa is often difficult to detect by CT due to beam-hardening artifacts, such as streak artifacts from the internal occipital protuberance or interpetrous lucency. ${ }^{17,18)}$ In other words, CT can detect fourth ventricle IVH, but not cisternal SAH, in most patients with ruptured distal PICA aneurysm. In contrast, FLAIR imaging is particularly useful for demonstrating acute cisternal SAH in the posterior fossa, such as the cerebellopontine angle cistern or cisterna magna, in patients with ruptured distal PICA aneurysm. In the present study, FLAIR imaging depicted both fourth ventricle IVH and cisternal SAH in the posterior fossa without artifacts in a patient with ruptured distal PICA aneurysm. Furthermore, MR angiography confirmed the aneurysm. We emphasize that FLAIR is extremely useful for the diagnosis of cisternal SAH in the posterior fossa.

In this study, all subjects had aneurysmal SAH. Therefore, we could not determine the false-positive or false-negative rates, likelihood ratios, or sensitivity, because no control subjects without SAH were included. Instead, we compared the ability to identify SAH or IVH using the frequency of undetected hematomas.

The present study showed that FLAIR imaging was more sensitive than CT for detecting acute SAH within 24 hours after onset, and the frequency of undetected SAH was higher for CT than for FLAIR imaging. However, the diagnostic accuracy of FLAIR imaging for the detection of acute $\mathrm{SAH}$ was reduced in comparatively tight cisterns, such as the interhemispheric cistern. Clinicians can overcome this drawback by simultaneously performing MR angiography. FLAIR imaging is likely to become the gold standard for the diagnosis of $\mathrm{SAH}$ as well as minor hemorrhages.

\section{References}

1) Atlas SW: MR imaging is highly sensitive for acute subarachnoid hemorrhage...not! Radiology 186: 319-323, 1993

2) Bakshi R, Caruthers SD, Janardhan V, Wasay M: Intraventricular CSF pulsation artifact on fast fluid-attenuated inversion-recovery MR images: analysis of 100 consecutive normal studies. AJNR Am J Neuroradiol 21: 503-508, 2000

3) Bakshi R, Kamran S, Kinkel PR, Bates VE, Mechtler LL, Janardhan V, Belani SL, Kinkel WR: Fluid-attenuated inversion-recovery MR imaging in acute and subacute cerebral intraventricular hemorrhage. 
AJNR Am J Neuroradiol 20: 629-636, 1999

4) Cianfoni A, Martin MG, Du J, Hesselink JR, Imbesi SG, Bradley WG, Bydder GM: Artifact simulating subarachnoid and intraventricular hemorrhage on single-shot, fast spin-echo fluid-attenuated inversion recovery images caused by head movement: A trap for the unwary. AJNR Am J Neuroradiol 27: 843-849, 2006

5) Dodick DW: Thunderclap headache. Headache 42: 309-315, 2002

6) Drake CG: Report of World Federation of Neurological Surgeons Committee on a universal subarachnoid hemorrhage grading scale. J Neurosurg 68: 985-986, 1988 (letter)

7) Ducros A, Boukobza M, Porcher R, Sarov M, Valade $\mathrm{D}$, Bousser MG: The clinical and radiological spectrum of reversible cerebral vasoconstriction syndrome. A prospective series of 67 patients. Brain 130: 3091-3101, 2007

8) Fisher CM, Kistler JP, Davis JM: Relation of cerebral vasospasm to subarachnoid hemorrhage visualized by computerized tomographic scanning. Neurosurgery 6: 1-9, 1980

9) Herlihy AH, Hajnal JV, Curati WL, Virji N, Oatridge A, Puri BK, Bydder GM: Reduction of CSF and blood flow artifacts on FLAIR images of the brain with kspace reordered by inversion time at each slice position (KRISP). AJNR Am J Neuroradiol 22: 896-904, 2001

10) Hijdra A, Brouwers PJ, Vermeulen M, van Gijn J: Grading the amount of blood on computed tomograms after subarachnoid hemorrhage. Stroke 21: 1156-1161, 1990

11) Jakobsson KE, Saveland H, Hillman J, Edner G, Zygmunt S, Brandt L, Pellettieri L: Warning leak and management outcome in aneurysmal subarachnoid hemorrhage. J Neurosurg 85: 995-999, 1996

12) Klimo P Jr, Schmidt RH: Computed tomography grading schemes used to predict cerebral vasospasm after aneurysmal subarachnoid hemorrhage: a historical review. Neurosurg Focus 21(3): E5, 2006

13) Lewis SB, Chang DJ, Peace DA, Lafrentz PJ, Day AL: Distal posterior inferior cerebellar artery aneurysms: clinical features and management. J Neurosurg 97: 756-766, 2002

14) Maeda M, Yagishita A, Yamamoto T, Sakuma $H$, Takeda K: Abnormal hyperintensity within the subarachnoid space evaluated by fluid-attenuated inversion-recovery MR imaging: a spectrum of central nervous system diseases. Eur Radiol 13 Suppl 4: L192-201, 2003

15) Mitchell P, Wilkinson ID, Hoggard N, Paley MN, Jellinek DA, Powell T, Romanowski C, Hodgson T, Griffiths PD: Detection of subarachnoid haemorrhage with magnetic resonance imaging. J Neurol Neurosurg Psychiatry 70: 205-211, 2001

16) Mohamed M, Heasly DC, Yagmurlu B, Yousem DM: Fluid-attenuated inversion recovery MR imaging and subarachnoid hemorrhage: not a panacea. AJNR Am J Neuroradiol 25: 545-550, 2004

17) Mostrom U, Ytterbergh C: Artifacts in computed tomography of the posterior fossa: a comparative phantom study. J Comput Assist Tomogr 10: 560-566, 1986

18) Noguchi K, Ogawa $T$, Inugami A, Toyoshima $H$, Sugawara S, Hatazawa J, Fujita H, Shimosegawa E, Kanno I, Okudera T: Acute subarachnoid hemorrhage: MR imaging with fluid-attenuated inversion recovery pulse sequences. Radiology 196: 773-777, 1995

19) Noguchi K, Ogawa T, Seto $H$, Inugami A, Hadeishi H, Fujita H, Hatazawa J, Shimosegawa E, Okudera T, Uemura K: Subacute and chronic subarachnoid hemorrhage: diagnosis with fluid-attenuated inversion-recovery MR imaging. Radiology 203: 257-262, 1997

20) Noguchi K, Seto H, Kamisaki Y, Tomizawa G, Toyoshima S, Watanabe N: Comparison of fluid-attenuated inversion-recovery MR imaging with CT in a simulated model of acute subarachnoid hemorrhage. AJNR Am J Neuroradiol 21: 923-927, 2000

21) Norman D, Price D, Boyd D, Fishman R, Newton TH: Quantitative aspects of computed tomography of the blood and cerebrospinal fluid. Radiology 123: 335-338, 1977

22) Okawara SH: Warning signs prior to rupture of an intracranial aneurysm. J Neurosurg 38: 575-580, 1973

23) Passero S, Ulivelli M, Reale F: Primary intraventricular haemorrhage in adults. Acta Neurol Scand 105: 115-119, 2002

24) Sames TA, Storrow AB, Finkelstein JA, Magoon MR: Sensitivity of new-generation computed tomography in subarachnoid hemorrhage. Acad Emerg Med 3: 16-20, 1996

25) Tsuchiya K, Inaoka S, Mizutani Y, Hachiya J: Fast fluid-attenuated inversion-recovery $M R$ of intracranial infections. AJNR Am J Neuroradiol 18: 909-913, 1997

26) van Gijn J, Hijdra A, Wijdicks EF, Vermeulen M, van Crevel H: Acute hydrocephalus after aneurysmal subarachnoid hemorrhage. J Neurosurg 63: 355-362, 1985

27) Vermeulen $M$, van Gijn J: The diagnosis of subarachnoid haemorrhage. J Neurol Neurosurg Psychiatry 53: 365-372, 1990

28) Woodcock RJ Jr, Short J, Do HM, Jensen ME, Kallmes DF: Imaging of acute subarachnoid hemorrhage with a fluid-attenuated inversion recovery sequence in an animal model: comparison with non-contrast-enhanced CT. AJNR Am J Neuroradiol 22: 1698-1703, 2001

29) Wu HM, Yousem DM, Chung HW, Guo WY, Chang CY, Chen CY: Influence of imaging parameters on high-intensity cerebrospinal fluid artifacts in fastFLAIR MR imaging. AJNR Am J Neuroradiol 23: 393-399, 2002

Address reprint requests to: Masami Shimoda, M.D., Department of Neurosurgery, Tokai University Hachioji Hospital, 1838 Ishikawa-machi, Hachioji, Tokyo 192-0032, Japan.

e-mail: mashimoda-nsu@umin.ac.jp 


\section{Commentary}

The authors describe the diagnostic efficacy of fluidattenuated inversion recovery (FLAIR) magnetic resonance imaging and computed tomography (CT) in patients with acute aneurysmal subarachnoid hemorrhage (SAH) within 24 hours after onset. This study showed that FLAIR imaging was more sensitive than CT imaging except for SAH in comparatively tight cistern, especially interhemispheric cistern. In their conclusion, the authors predict that FLAIR imaging may be the gold standard for the diagnosis of SAH.

CT is the initial diagnostic imaging modality of choice to evaluate for SAH and its sensitivity during the first 12 hours after SAH is known to be between $98 \%$ and $100 \%$. However, it declines to $93 \%$ at 24 hours and continues to decline after 24 hours. Some other studies have suggested that FLAIR imaging may be more sensitive and useful than CT in the subacute phase.

I think CT still remains the front line in SAH imaging because it is readily available, rapid, cost-effective, and easily-performed, particularly in critically ill patients, in spite of lower accuracy in the posterior fossa due to beam-hardening artifacts. This study is well analyzed semiquantitatively the efficacy of FLAIR imaging for detecting acute aneurysmal SAH within 24 hours in each cisterns and ventricles. FLAIR image, if validated by other well-designed prospective studies, has the potential to become the gold standard in detecting SAH. But, FLAIR imaging may be a supplementary diagnostic modality like lumbar puncture and it is still controversial and questionable that FLAIR imaging is likely to become the gold standard for the diagnosis of SAH.

Dae Hee Han, M.D., Ph.D. Department of Neurosurgery Director Cerebral and Cardiovascular Disease Center Professor Emeritus
Seoul National University Seoul, R.O.K.

The authors investigated 81 patients without rerupture of cerebral aneurysm who underwent both FLAIR imaging and CT at the time of admission and compared the findings of FLAIR MR imaging and CT in patients with acute-phase SAH to investigate the problems with SAH diagnosis by FLAIR imaging. CT has been considered to be the gold standard for the diagnosis of SAH. However, CT is unreliable for detecting subarachnoid blood produced by a minor leak. There are some cases with sudden onset of severe headache without SAH findings on CT. How to establish or exclude a diagnosis of $\mathrm{SAH}$ in these cases is very important. Conventionally, most neurosurgeons performed lumbar puncture to confirm the diagnosis of SAH. However, lumbar puncture carries the risk of rerupture of aneurysm in patients with SAH.

This study showed that FLAIR imaging was more sensitive than CT for detecting acute SAH within 24 hours after onset, and the frequency of undetected SAH was higher for CT than for FLAIR imaging. However, the diagnostic accuracy of FLAIR imaging was reduced in tight cisterns. The authors found that this disadvantage of FLAIR imaging can be supplemented by MR angiography performed simultaneously.

This very important study suggests that FLAIR imaging is more sensitive than CT for detecting acute SAH within 24 hours after onset, and the frequency of undetected SAH is higher for CT than for FLAIR imaging. FLAIR imaging may reduce the risk of rebleeding of patients with aneurysm who suffer sudden onset of severe headache without SAH findings on CT.

Yuichi TANAKA, M.D.

Department of Neurosurgery Saitama Medical Center Jichi Medical University Saitama, Saitama, Japan 